\title{
Distribution System State Estimation Using Compressive Sensing
}

M. Majidi ${ }^{\text {a }}$, M. Etezadi-Amoli *a ${ }^{*}$ H.Livani a 
Abstract- This paper leverages the big data provided by micro-phasor measurement units ( $\mu$ PMUs) placed along the smart distribution networks for distribution system state estimation (DSSE). We propose a novel and straightforward DSSE algorithm by solving a set of linear equations without any iterative process. The $\mu$ PMUs are placed at few buses to measure the voltage phasors. The measured voltage vector is expressed as the product of current injections vector and impedance matrix of the system. Since number of $\mu$ PMUs is less than number of buses, the constructed set of linear equations are underdetermined. Furthermore, the injection currents vector is sparse because any single load/generator current is negligible when compared with the total current injected from the external grid to the distribution network. Subsequently, we use Compressive Sensing and $\ell^{1}$-norm minimization to recover the sparse current vector from the limited number of $\mu$ PMUs. The voltages at all buses are obtained by multiplying the reconstructed current vector by the impedance matrix. Performance of our method is demonstrated on the IEEE 123-bus test system and a 13.8-kV, 134-bus real network with different distributed generations (DGs) penetration level and under a weakly meshed operation mode. Also, the performance of the proposed technique is compared with that of the conventional weighted least-square (WLS) method.

Keywords- State estimation; distribution networks; micro-phasor measurement units ( $\mu P M U s)$; $\ell_{1}$-norm minimization; compressive sensing (CS); $\ell^{1}$-regularized least square problem.

\section{INTRODUCTION}

State estimation (SE) for power transmission systems was first addressed by 
Schweppe in 1970s by solving a set of nonlinear equations using iterative weighted least square (WLS) algorithm [1]. SE has been firmly tailored to transmission systems and prevalently used at the control centers. However, distribution system state estimation (DSSE) is a formidable task due to peculiarities of distribution networks and limitations that are encountered.

DSSE was first proposed by Wu in 1990 [2], and enhanced by Roytelman in 1993 [3]. In [4], the Newton-Raphson method is used to solve a three-phase DSSE. In [5], [6], a current-based DSSE method is developed with the branch currents measured by current transformers. In [7], three-phase current values are similarly used to perform SE with a fast decoupled method. In [8], [9], DSSE is formulated by utilizing the information of the energy bills as the pseudo measurements. In [10], [11], a weighted least squares (WLS) state estimator is robustly built by the solutions of Normal equation and machine learning techniques to identify the voltage unbalance in the networks. Reference [12] takes advantage of radial property of distribution networks to alleviate the computational complexity of optimization problems in DSSEs. In [13], the radial feature of distribution networks is also exploited to propose a single- and three-phase DSSE method based on system quasi-symmetric matrix and voltage drop equations. The proposed DSSE methods are mostly based on iterative algorithms using either nonlinear optimization methods or load flow calculations. However, we solve a set of linear equations to estimate the voltage magnitudes and angles in the distribution network.

Furthermore, the high execution time and big database requirements for large number of states in distribution networks are partially improved by multi area SE (MASE) algorithms [14]. The network needs to be divided into subareas and all the measured 
data are shared between the subareas. Thus, a micro control center in each subarea and strong communication infrastructure are needed. However, our proposed algorithm does not need several control centers and high bandwidth communication infrastructure.

In the existing DSSE approaches, pseudo measurements need to be added to the set of measurements to ensure the observability of the distribution network. However, there exist high uncertainties in the pattern of power injections in distribution buses. Therefore, load forecasting is generally assigned as a sub-problem of DSSE algorithms to yield the load data as the pseudo measurements for these methods [15]. In this respect, several studies have been performed to model the loads by artificial intelligence (AI) techniques such as artificial neural networks (ANN), fuzzy logics, and adaptive nonlinear auto-regressive exogenous (NARX) [16]-[18]. In [19], heuristic methods are deployed based on load flow calculation and machine learning concepts for load allocation. In [13], it is assumed that pseudo measurements and their corresponding error weights are obtained from statistical and historical data of load profiles. However, the estimated load values deteriorate the SE performance due to the high variance estimates of actual load patterns. Also, the estimated loads are not synchronized with other real time measurements that affect the SE process.

In [16], ANN is used to derive a nonlinear function between reliable measurements and injection powers at the load buses. However, the trained neural network can not estimate the pseudo measurements accurately if reconfigurations happen in distribution networks. Therefore, the ANN needs to be retrained and saved. This imposes large data storage requirement for the data processing system. In [20], WLS estimator and threephase load flow developed based on Fortescue transformation are exploited to estimate 
the loads in large scale asymmetric distribution networks. In [21], a novel multi-phase DSSE technique is introduced to address the implementation details of a constrained WLS method by incorporating industrial-grade modeling of system components into a standard three-phase state estimator. Unlike the existing DSSE, our proposed algorithm does not rely on pseudo measurements with high uncertainties and low accuracy. To get a reliable solution from WLS-based DSSE methods, we need to tune the error weight of several pseudo measurements by trial and error. However, we don't need to do this type of extensive studies in our proposed algorithm.

Since the synchronized measured data by micro-phasor measurement units ( $\mu$ PMUs) with high sampling rate can provide remarkable accuracy in different applications of distribution management systems (DMS) especially in fault detection, isolation and restoration (FDIR) and DSSE [22]-[25], smart distribution networks will be inevitably equipped with $\mu$ PMUs in the near future. However, since PMUs are generally used in the power transmission networks, many projects have been assigned to introduce the applications and address the challenging issues of $\mu$ PMUs in distribution networks [26]. The use of synchronized measurements for efficient distribution system state estimation algorithms has been discussed in [27]-[33]. Currently in [34] a new research on phasor measurement accuracy in a real power grid environment at the distribution level has been defined. Regular measurements and $\mu \mathrm{PMU}$ data are transmitted to distribution system control centers every few seconds while this rate for smart meters recording the power injections (loads and generations) is in the range of few minutes. Thus, there are challenges regarding the synchronization and different reporting rates of these devices which are discussed in [35]. However, we only use the measured data by a few $\mu$ PMUs 
along the distribution network and do not need to consider the challenges associated with different reporting rates of measurement devices.

In this paper, we propose a novel, three-phase, and $\mu$ PMU-based DSSE paradigm by utilizing the measured voltage phasors at few buses and the impedance matrix of the system. We effectively exploit $\ell^{1}$-norm minimization methods and compressive sensing (CS) technique to estimate the three-phase current injections vector by solving an underdetermined linear equation system. Also, we propose a new method to determine the regularization parameter in $\ell^{1}$ regularization techniques. After including the impedance of external grid to the system impedance matrix, voltages are calculated by multiplying the impedance matrix and the sparse injection currents vector. We then cast the DSSE problem to a sparse vector reconstruction problem and estimate the sparse injection currents vector. Given the currents vector and impedance matrix of the system, voltage magnitudes and angles are simply calculated for all buses in the network.

CS is an emerging signal processing technique whose applications in power systems have been disclosed in [24], [25], [36]-[45]. In [24], [25], CS is used for locating the single and simultaneous faults in distribution networks by adopting different optimization techniques. In [36], CS is used in the sparse representation classifier for partial discharge patterns recognition. Reference [37] reduces the amount of aggregated power information for DSSE using CS. In [38], the greedy orthogonal matching pursuit (OMP) method and the least-absolute shrinkage and selection operator (Lasso) are used to identify multiple line outages in power systems. One recent study in [39] uses Lasso formulation for identifying and pinpointing the faults in power systems. In [40]-[44], applications of CS in the power system topology identification are discussed. Also, the 
performance of the sparse recovery models for bad data analysis and power system state estimation is investigated in [45].

The rest of the paper is organized as follows. Section II describes our proposed state estimation algorithm using CS technique and $\ell^{1}$-norm minimization. The simulation results are detailed in Section III to evaluate the performance of the proposed method in different distribution operation scenarios under most operating conditions. The features of our proposed DSSE algorithm are discussed in Section IV. Conclusions and future work are presented in section $\mathrm{V}$.

\section{STATE ESTIMATION METHODOLOGY}

Due to heterogeneity of overhead lines in distribution networks, positive-, negative-, and zero-sequence of overhead line impedances are coupled together and solving each circuit separately does not produce accurate results [24], [25]. Therefore, transforming the three-phase voltages and currents to symmetrical components is not beneficial and we use the three-phase system model to formulate our DSSE algorithm.

In an N-bus distribution network, voltage at each bus is expressed as a linear combination of the injection currents at all the buses

where is the three-phase voltage vector including sub-vectors denoting phases to ground voltages at bus . is the three-phase impedance matrix of the system made of sub-matrices and is the current injections vector formed by sub-vectors displaying phases current injections at bus .

The is calculated by three-phase impedance and admittance matrices of overhead 
lines and three-phase impedance matrix of the remote-terminal system. Each line is represented by a three-phase $\pi$-model as illustrated in Fig. 1 .

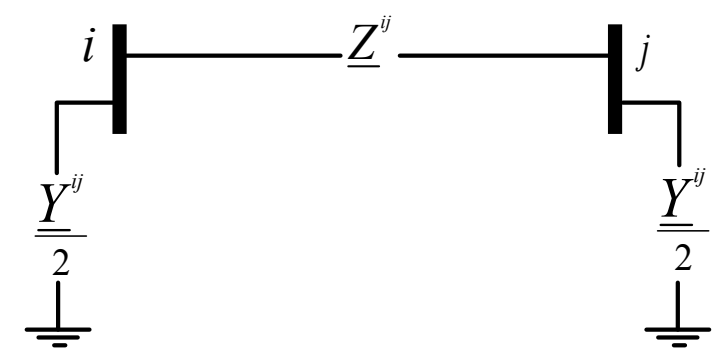

Fig. 1. Three-phase $\pi$-model of overhead lines

where ,.

Thevenin impedance of the external grid is included in the impedance matrix of the system by adding the impedance between the beginning bus of the feeder and ground. This impedance is much smaller than the shunt impedances between any bus and ground [46]. Therefore, the injected current to the remote-terminal is much larger than those in other buses. In equation (1), if we assume that bus \# 1 is connected to the external grid, . Subsequently, the current vector can be treated as a sparse vector with one dominant term and many negligible entries. Since, it is not feasible to monitor the voltage phasor for all buses, we only measure three-phase voltage magnitudes and angles at buses by $\mu$ PMUs and utilize them as vector. We then select the rows of corresponding to the measurement buses and concatenate them to form . Now, vector can be expressed as a product of and

Since coefficients of and in (2) are complex values, we rewrite (2) in terms of their real (Re) and imaginary (Im) parts as 
By separating the real and imaginary equations

Equations (4) and (5) are written in a matrix format as

where, . Since the number of equations is less than number of unknowns, (6) is an underdetermined set of equations with several different feasible solutions. In this paper, we utilize compressive sensing (CS) and $\ell^{1}$-norm minimization techniques to efficiently acquire the sparse vector via very few measurements ( and a basis matrix ( in the underdetermined linear system. Based on Shannon-Nyquist sampling theorem, a signal is exactly reconstructed if it is captured by twice the highest signal frequency which is the so-called Nyquist sampling rate. However, the huge amount of the generated samples in the Nyquist rate for several emerging applications e.g. imaging make the signal acquisition and processing too costly and even impossible. Therefore, these logistical challenges inspire the CS to reconstruct the sparse representation of the dense signals in a transform domain from far fewer samples than needed by Nyquist theorem [47]. Since it is not expected that $\mu$ PMUs are installed at all buses in distribution networks and the bus voltages vector can be related to a sparse injection currents vector through the impedance matrix as the basis, CS is well-suited for estimating the voltage phasors at all buses.

In order to find the sparsest solution of vector satisfying equation (6), we need to 
minimize the $\ell^{0}$-norm of

where is norm- operator. Although solving $\ell^{0}$-norm minimization is NP-hard problem or computationally intractable, it is proved that $\ell^{1}$-norm minimization can accurately approximate the solution of equation (7) if the vector is sparse enough [47]

The injection current vector from the remote bus is much higher than the injection current for the load/generator buses. Therefore, the injection currents vector is not completely sparse. We overcome this technical difficulty by exploiting the $\ell^{1}$ regularized least square problem (LSP) solvers that suitably reconstruct the approximate sparse vectors by solving [48]

where is the regularization parameter.

Now, the voltage magnitudes and angles in all the buses are obtained by multiplying the estimated injection currents vector by the impedance matrix in equation (1). The $\ell^{1}-$ regularized (LSP) methods estimate the significant injection current entry in the remote bus along with considerable small values associated with big loads and generators.

Theorem 1. The $\ell^{1}$ regularization methods reconstruct the sparse signals with nonzero values with high probability if

where is a small value. For example, gives a probability of decoding failure of less than $0.15 \%$ [47].

In theorem 1, we need to have a priori knowledge of nonzero values in the sparse 
vector. In the developed DSSE problem, there are three dominant nonzero values corresponding to three-phase injected currents to the remote-terminal bus. As a generalized $\mu$ PMU placement scheme, $\mu$ PMUs must be particularly installed at the remote-terminal bus and those buses with large loads, shunt capacitors, and DGs whose injection currents are great.

The $\ell^{1}$-regularized (LSP) (9) cannot be analytically solved and consequently several numerical algorithms have been proposed to solve that. In [49], the ellipsoid or subgradient methods are introduced to solve (9). Also, standard interior-point methods for solving the small/medium size problems [50] and customized interior-point methods for handling the large scale problems are proposed in [51]. In addition, homotopy methods and variants are currently used to solve (9) [52]. In this paper, we adopt a specified interior-point method for solving (9) [48]. The $\ell^{1}$-regularized least square problem method efficiently solves the underdetermined set of linear equations with thousands of variables in a couple of seconds [48].

\section{Optimality Conditions and Tuning the Regularization Parameter}

Since the objective function of (9) is convex but not differentiable, a first-order optimality condition is used to obtain the sufficient optimal conditions of for (9)

The optimal solution of (9) is obtained when which implies, where . In addition, must not run all the way to zero. Therefore, an upper bound on is defined as, where [53].

Several iterative methods have been proposed to determine the regularization parameter based on the sparsity level of the recovery signal or measurement noise 
variance [38], [53]. In this paper, we propose a new method to quantify the based on the regularization path.

Theorem 2. The regularization path of the $\ell^{1}$-regularized (LSP), i.e., the family of solutions as varies over has the piecewise-linear solution path property: There are values, with, such that the regularization path is a piecewise linear curve

where solves (9) with, and is the solution of (9).

Using theorem 2, the solution can be linearly related to by

Parameters and are computed by selecting any two values and, with, and calculating their corresponding solutions and by (9). Then, varies over and the is obtained by (11) and substituted in (9) to find the objective function values as

The value for that minimizes the objective function is selected for the problem.

\section{SIMULATION RESULTS}

To evaluate the accuracy and the performance of the proposed algorithm, a real 13.8$\mathrm{kV}, 134-$ bus distribution network and the IEEE 123-bus test system are considered. The networks are simulated using DIgSILENT Power Factory software [54] and the threephase unbalanced load flow results are exported to MATLAB for processing. The threephase voltage magnitudes and angles that are measured at few buses are fed to our MATLAB algorithm. We evaluate the accuracy of the proposed algorithm by calculating 
the mean absolute error (MAE) index for the estimated voltage magnitudes and angles as

where, , are estimated and actual three-phase voltage phasors at bus, respectively, and is number of non-monitored buses (.

\section{13.8-kV, 134-Bus Network}

Single line diagram of the 134-bus system is shown in Fig. 2. Impedance matrices of the heterogeneous overhead lines and load information are adopted from [24]. In addition, we incorporate the shunt admittance matrices of each line type into our modeling.

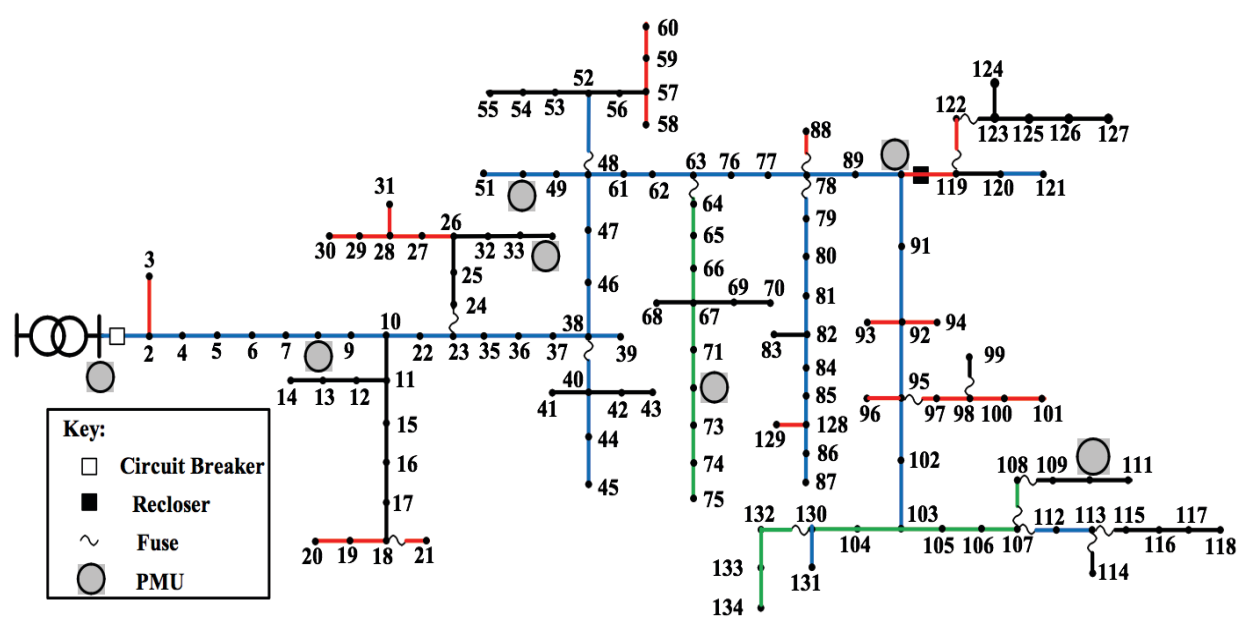

Fig. 2. Single line diagram of the test system

Based on theorem 1, for and,. Therefore, we assume that $7 \mu$ PMUs are placed at buses \# 1, 8, 34, 50, 72, 90, and 110 based on the described generalized measurement placement. Using the proposed method, a value of $10^{-4}$ is obtained for in this problem. 
For the 134-bus test system, and. Therefore, and are selected from the range of and (9) is solved for each to calculate their corresponding solutions and . Using (11), parameters and are calculated and placed in (12). The value increases by a step size of for the range of in (12) and the objective function () is calculated. Therefore, the minimum objective function value is obtained with. The objective function (versus is shown in Fig. 3.

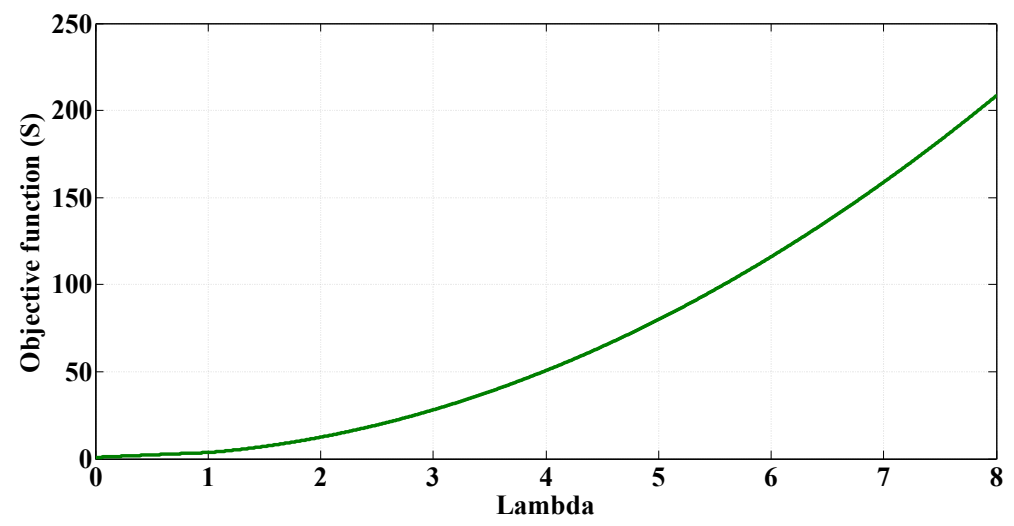

Fig. 3. Objective function ( versus for the selection process

Although, voltage phasors in adjacent buses to $\mu$ PMUs can be calculated by the measured currents through incident branches to the $\mu$ PMU buses, we assume that $\mu$ PMUs only measure the voltage phasors at buses and not the incident branch currents. Clearly, more measured voltage data would result in better state estimation.

Actual and estimated injection currents are shown in Fig. 4. We use the load flow voltage phasors in all buses to calculate the actual injection currents . 


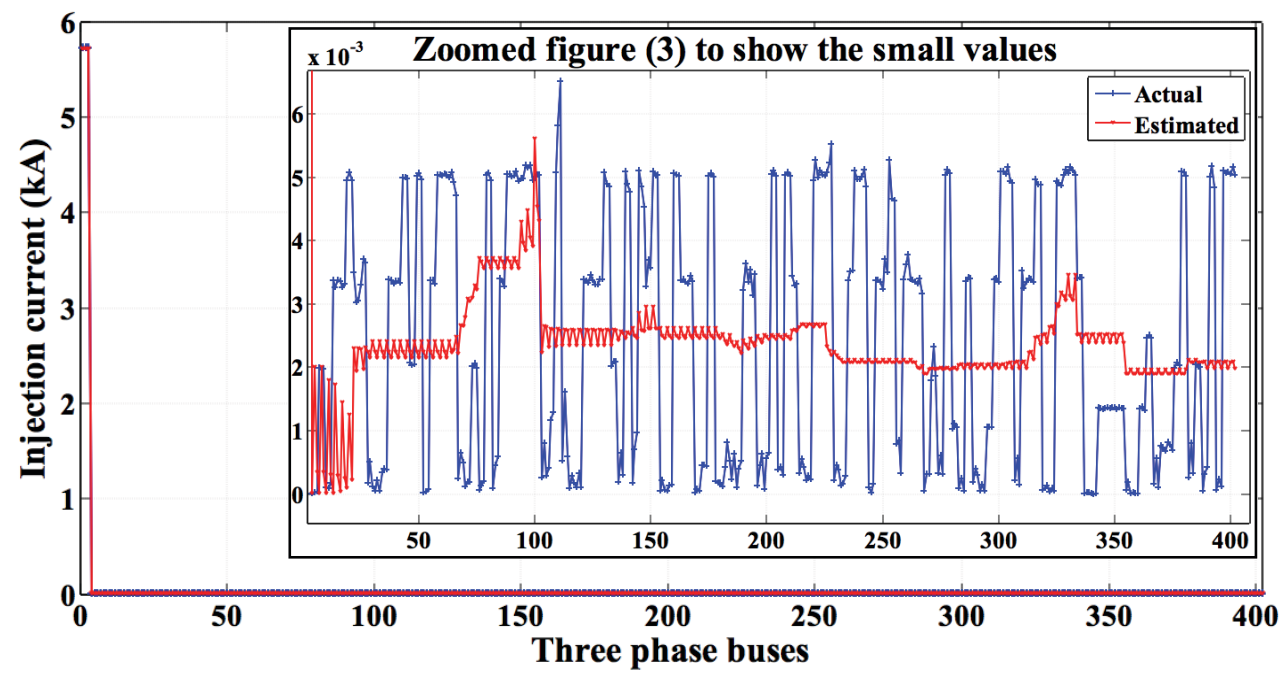

Fig. 4. Actual and estimated injection currents in the 134-bus system

There are 402 phases in the system since all 134 buses are three phase buses. As shown, the current vector is sparse with three dominant values in the range of kilo ampere corresponding to three phases in bus \# 1 and small values in the range of ampere corresponding with three phases of other buses. The estimated and actual threephase voltage magnitudes for all buses are shown in Fig. 5. Also, the estimated and actual voltage angles at all buses for phase (a) are presented in Fig. 6. As shown, the proposed method has satisfactory performance and the estimated voltage magnitudes and angles have good correlation with the actual voltage magnitudes and angle.

Since the proposed state estimation equation is an underdetermined equation, the current solution is not unique and large estimation errors may be obtained. However, $\ell^{1}-$ regularized least square method solves the injection current vector by minimizing the estimation errors of voltage phasors at the measurement buses. Also, there is a high correlation among the voltage phasors along the distribution feeders. Therefore, when the voltage phasors at few measurement buses are accurately estimated, voltage phasors at other buses are also accurately calculated via multiplying the impedance matrix by 
the estimated current vector.

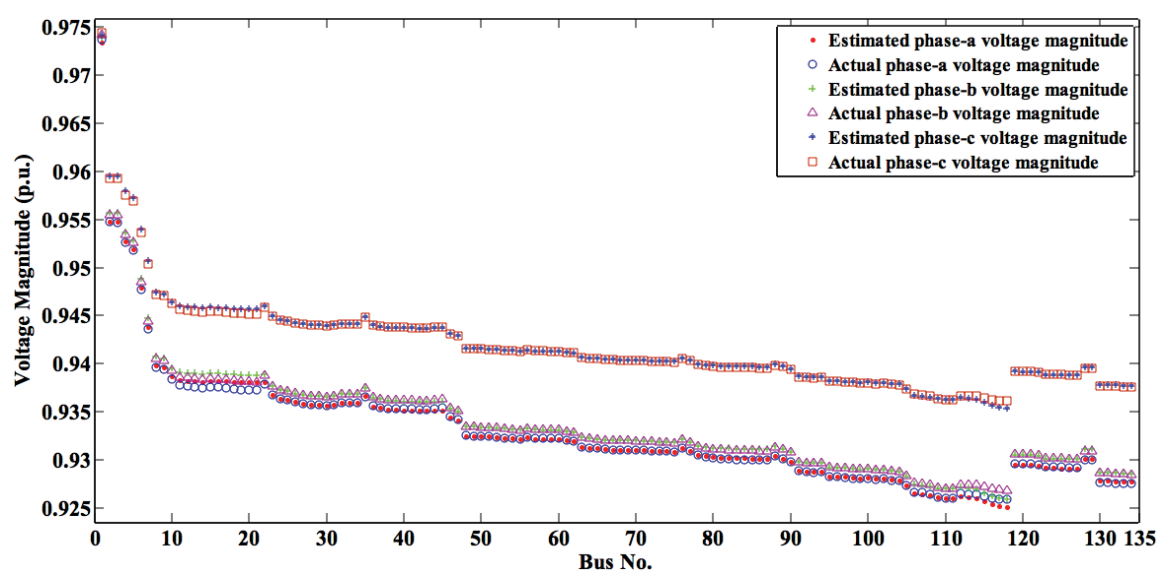

Fig. 5. Actual and estimated three-phase voltage magnitudes (p.u.) at all buses

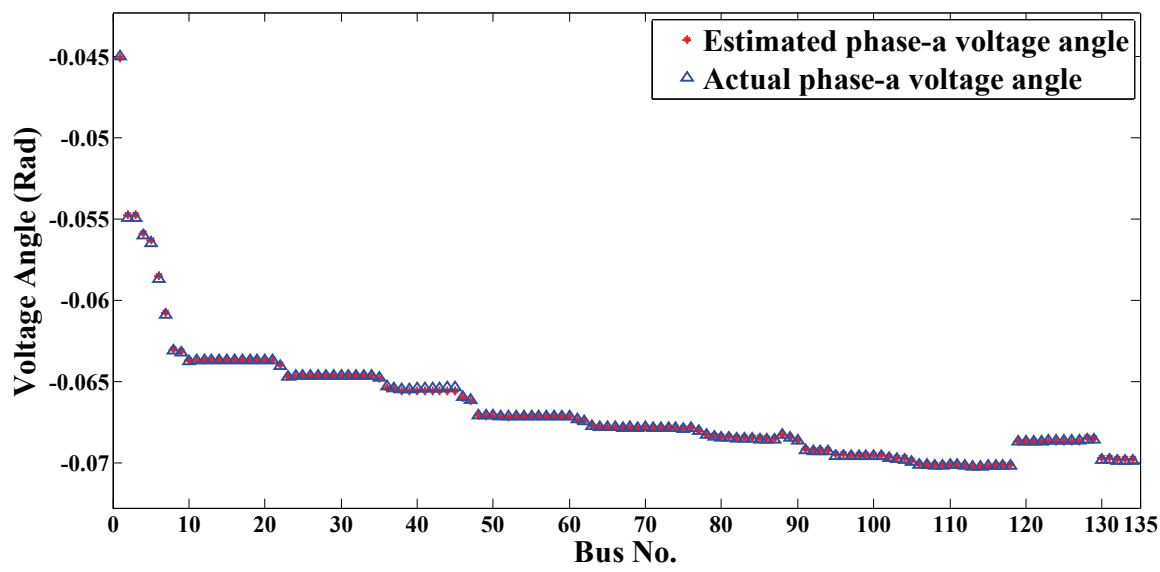

Fig. 6. Actual and estimated three-phase voltage angles (Rad) at all buses

The voltage magnitude and angle estimation errors are shown in Fig. 7 and 8, respectively. 


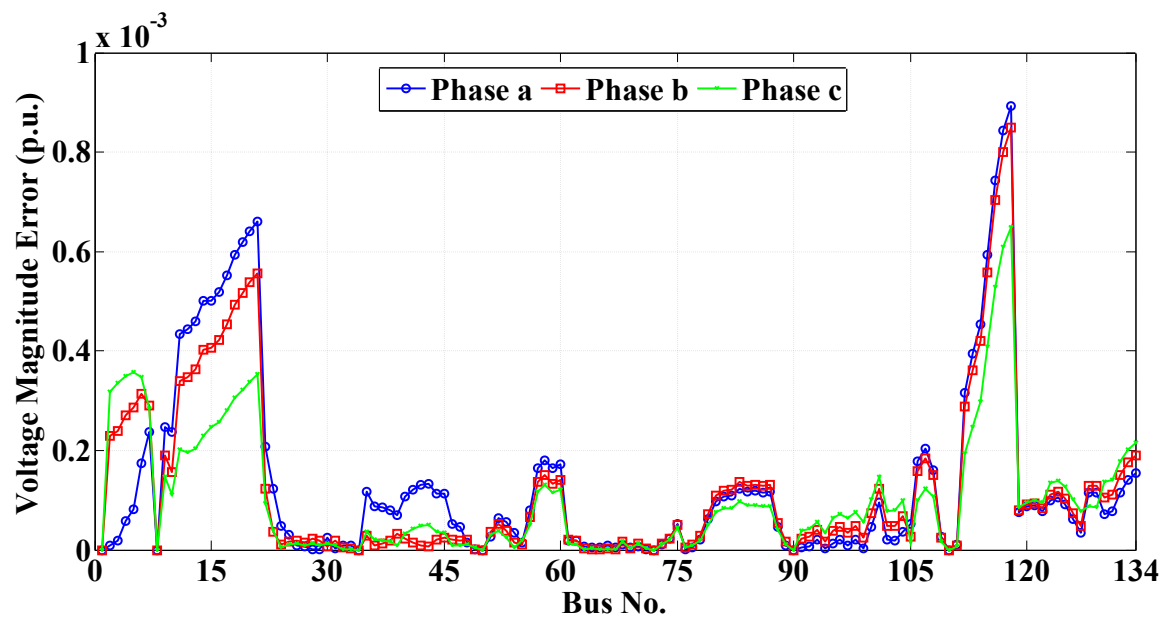

Fig. 7. Voltage magnitude estimation errors (p.u.) at all buses

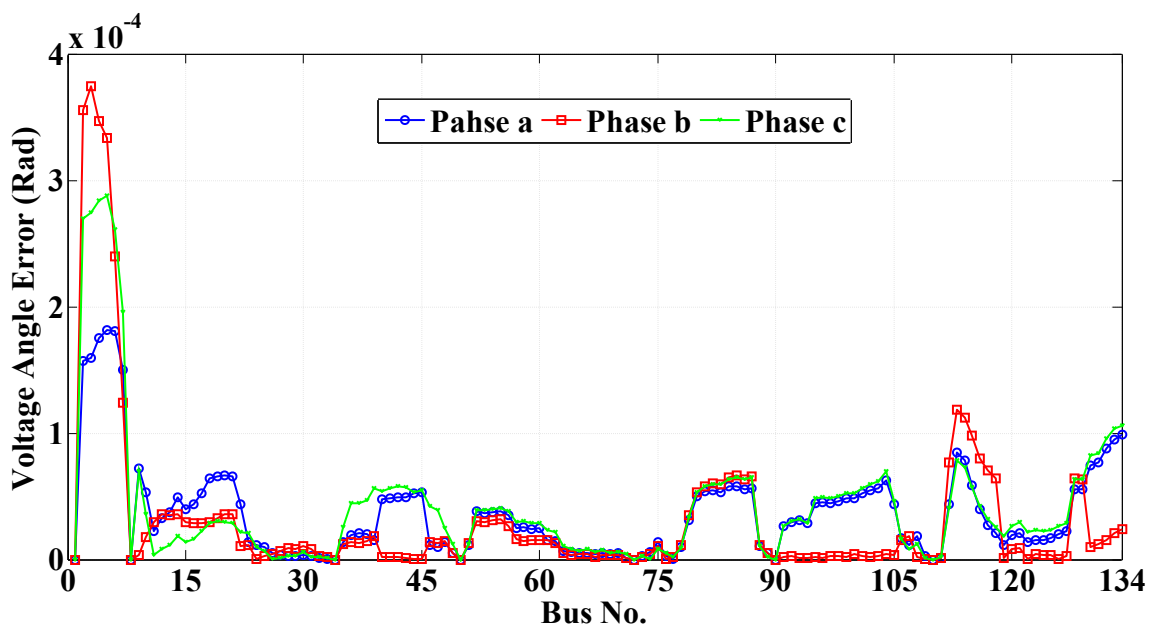

Fig. 8. Voltage angle estimation errors (p.u.) at all buses

In order to evaluate the performance of the method statistically, the histograms of the voltage magnitude and angle estimation errors are shown in Fig. 9. Most of the voltage angle estimation errors are in the range of. The voltage magnitude estimation errors are in the range of and of the estimated states are calculated and in this scenario, respectively.

The proposed DSSE algorithm is executed on a computer with a $2.4-\mathrm{GHz}$ Intel Core i3-2370 M CPU and 4.00-GB memory and the execution time is 3 seconds. 


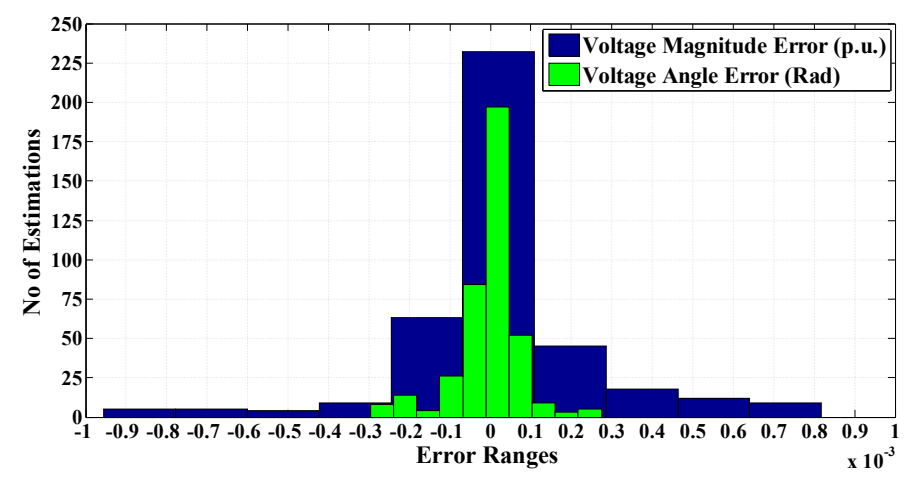

Fig. 9. Histograms of voltage magnitude and angle estimation errors

\subsection{Noisy Measurements Assessment}

Although $\mu$ PMUs are much more accurate than pseudo measurements, they are usually influenced by both measurement and communication errors. Generally, measured voltage magnitudes are corrupted by the voltage measurement error and measured voltage angles are erroneous due to synchronization error.

Based on [55], the measurement errors in distribution PMUs are in the range of $0.1 \%$ and the synchronization error is $100 \mathrm{~ns}$ that is equivalent to in a $50 \mathrm{~Hz}$ system. We incorporate higher level of errors to the simulated measurement data by multiplying each phase to ground voltage data by, where are randomly generated from a zero-mean normal distribution with standard deviation $0.1 \%$ and $0.01^{\circ}$, respectively. In this case, and of the estimations are and, respectively. The histograms of the voltage magnitude and angle estimation errors are depicted in Fig. 10 for the state estimation with noisy measurements. Compared to ideal $\mu$ PMU measurements, our algorithm produces a slightly reduced accuracy with satisfactory results under noisy measurements.

As shown in Fig. 10, most of the voltage magnitude errors are in the range of and most of the voltage angle errors are in the range of. 


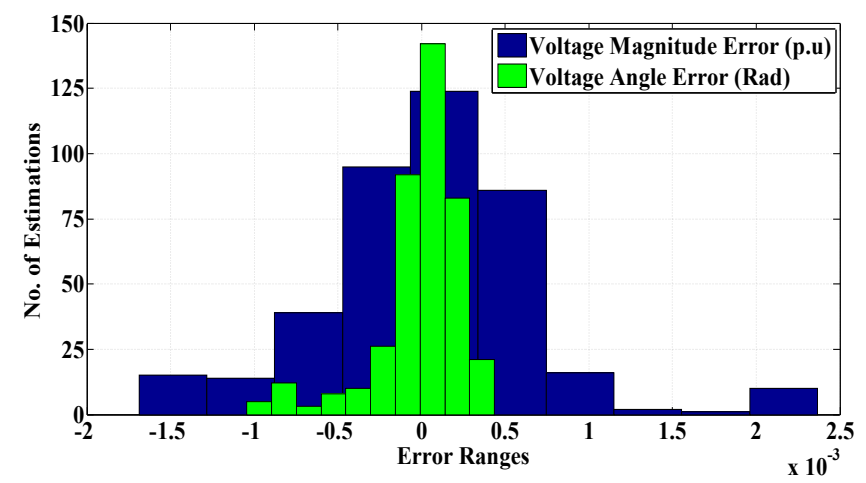

Fig. 10. Histograms of voltage phasor estimation errors using noisy measurements

The estimation accuracy and run time for the proposed DSSE are dependent on the measurement noise level. To show this issue, the simulated PMU data are corrupted by 2-8 orders of magnitude larger than the standard errors of the $\mu$ PMUs. The average , and run time over the 50 simulation runs at each noise level are presented in Table I.

TABLE I. State Estimation PeRFoRMANCE IN DIFFERENT NOISE LEVELS

\begin{tabular}{c|c|c|c|c}
\hline \multicolumn{2}{c|}{ Noise level } & $\begin{array}{c}\text { Average/Maximum } \\
(\text { p.u. })\end{array}$ & $\begin{array}{c}\text { Average/Maximum } \\
\times 10^{-4}\end{array}$ & $\begin{array}{c}\text { Average } \\
\times 10^{-4}\end{array}$ \\
\hline$(\%)$ & $\left({ }^{\circ}\right)$ & $3.2 / 5.12$ & $1.2 / 1.92$ & 2.7 \\
\hline 0.1 & 0.01 & $5.5 / 8.38$ & $2.1 / 3.1$ & 5.1 \\
\hline 0.2 & 0.02 & $7.5 / 12$ & $2.7 / 3.88$ & 5.6 \\
\hline 0.3 & 0.03 & $8.7 / 14$ & $3.2 / 4.67$ & 6.2 \\
\hline 0.4 & 0.04 & $10.8 / 18$ & $3.6 / 6.2$ & 5.9 \\
\hline 0.5 & 0.05 & $12.1 / 18$ & $4.2 / 5.58$ & 6.2 \\
\hline 0.6 & 0.06 & $14.7 / 23$ & $4.4 / 6.5$ & 5.8 \\
\hline 0.7 & 0.07 & $16.6 / 28$ & $4.8 / 7.1$ & 6.2 \\
\hline 0.8 & 0.08 & &
\end{tabular}

\subsection{DGs Penetration Level Assessment}

Distributed generations (DGs) penetration level has been increasing in smart 
distribution networks which lead to new technical challenges. In some cases, DGs not only supply the total load of a distribution feeder, but also inject extra generation into the external grid. In order to evaluate the performance of the proposed DSSE in the presence of DGs, various scenarios with different DG levels and locations as listed in Table II are considered.

As shown in Table II, the mean absolute errors (MAEs) of the estimated voltage magnitudes and angles do not increase significantly as DGs penetration level increases in the distribution network. Both and values are in the range of. Thus, the proposed DSE algorithm has an acceptable accuracy level in distribution networks with different DGs penetration levels.

Table II. State Estimation Performance in Different DGs Penetration LeVels

\begin{tabular}{|c|c|c|c|c|c|c|c|c|c|c|}
\hline $\begin{array}{c}\text { Sce } \\
\text { nar } \\
\text { io } \\
\text { No. }\end{array}$ & $\begin{array}{l}\mathrm{N} \\
\text { o. } \\
\text { of } \\
\mathrm{D} \\
\text { Gs }\end{array}$ & $\begin{array}{c}\text { Bus } \\
1 \\
\text { MW } \\
1 \\
\text { p.f. } \\
1\end{array}$ & $\begin{array}{c}\text { Bus } \\
2 \\
--- \\
\text { MW } \\
2 \\
-- \\
\text { p.f. } \\
2\end{array}$ & $\begin{array}{c}\text { Bus } \\
3 \\
-- \\
\text { MW } \\
3 \\
-- \\
\text { p.f. } \\
3\end{array}$ & $\begin{array}{c}\text { Bus } \\
4 \\
---- \\
\text { MW } \\
4 \\
--- \\
\text { p.f. } \\
4\end{array}$ & $\begin{array}{c}\text { Bus } \\
5 \\
--- \\
\text { MW } \\
5 \\
-- \\
\text { p.f. } \\
5\end{array}$ & $\begin{array}{c}\text { Bus } \\
6 \\
\text { MW } \\
6 \\
\text { p.f. } \\
6\end{array}$ & $\begin{array}{c}\text { Bus } \\
7 \\
--- \\
\text { MW } \\
7 \\
--- \\
\text { p.f. } \\
7\end{array}$ & $\times 10^{-4}$ & $\begin{array}{c}\times 10^{-} \\
4\end{array}$ \\
\hline 1 & 2 & $\begin{array}{l}45 \\
-0.5 \\
--- \\
0.8\end{array}$ & $\begin{array}{l}24 \\
-0.4 \\
-0.8\end{array}$ & & & & & & 1.63 & .469 \\
\hline 2 & 3 & $\begin{array}{l}45 \\
--- \\
0.5 \\
---- \\
0.8\end{array}$ & $\begin{array}{l}24 \\
--4 \\
0.4 \\
--8\end{array}$ & $\begin{array}{c}65 \\
----1 \\
--- \\
-0.8\end{array}$ & & & & & 1.86 & .115 \\
\hline
\end{tabular}




\begin{tabular}{|c|c|c|c|c|c|c|c|c|c|c|}
\hline 3 & 4 & $\begin{array}{c}45 \\
--- \\
0.5 \\
--- \\
0.8\end{array}$ & $\begin{array}{c}24 \\
---4 \\
0.4 \\
0.8\end{array}$ & $\begin{array}{c}65 \\
--- \\
1 \\
-0.8\end{array}$ & $\begin{array}{c}53 \\
--- \\
1 \\
-- \\
0.8\end{array}$ & & & & 3.29 & 1.04 \\
\hline 4 & 5 & $\begin{array}{c}45 \\
--5 \\
0.5 \\
0.8\end{array}$ & $\begin{array}{c}24 \\
0.4 \\
0.8\end{array}$ & $\begin{array}{c}65 \\
-- \\
1 \\
---8\end{array}$ & $\begin{array}{c}53 \\
-- \\
1 \\
---8 \\
0.8\end{array}$ & $\begin{array}{c}102 \\
--- \\
1 \\
--1 \\
0.8\end{array}$ & & & 3.28 & 1.92 \\
\hline 5 & 6 & $\begin{array}{c}45 \\
---- \\
0.5 \\
--- \\
0.8\end{array}$ & $\begin{array}{c}24 \\
-- \\
0.4 \\
-- \\
0.8\end{array}$ & $\begin{array}{c}65 \\
--- \\
1 \\
--- \\
0.8\end{array}$ & $\begin{array}{c}53 \\
--- \\
1 \\
---8 \\
0.8\end{array}$ & $\begin{array}{c}102 \\
---- \\
1 \\
--- \\
0.8\end{array}$ & $\begin{array}{c}85 \\
---- \\
1 \\
---- \\
1\end{array}$ & & 3.83 & 1.31 \\
\hline 6 & 7 & $\begin{array}{c}45 \\
-0.5 \\
--- \\
0.8\end{array}$ & $\begin{array}{c}24 \\
-0.4 \\
--8 \\
0.8\end{array}$ & $\begin{array}{c}65 \\
-1 \\
-- \\
0.8\end{array}$ & $\begin{array}{c}53 \\
-- \\
1 \\
---8 \\
0.8\end{array}$ & $\begin{array}{c}102 \\
--- \\
1 \\
0.8\end{array}$ & $\begin{array}{c}85 \\
--- \\
1 \\
--- \\
1\end{array}$ & $\begin{array}{c}127 \\
-- \\
1 \\
--- \\
1\end{array}$ & 5.02 & 2.56 \\
\hline
\end{tabular}

\subsection{Weakly Meshed Operation Mode Assessment}

Radial distribution networks are temporarily operated in a weakly meshed configuration due to fault isolation and load shifting of a feeder to its neighboring feeders. We simulate this condition in our test case by connecting the bus \# 85 to 72 by a switch in the presence of seven DGs of scenario 6. The mean absolute error of voltage magnitudes and angles are and, respectively. So, the weakly meshed operation mode along with the high DGs penetration level does not affect the performance of our state estimation algorithm. The histograms of the voltage magnitude and angle estimation errors are shown in Fig. 11. It is observed that the range of the voltage magnitude and angle estimation errors is not changed notably even in the meshed operation mode with high DGs penetration level. 


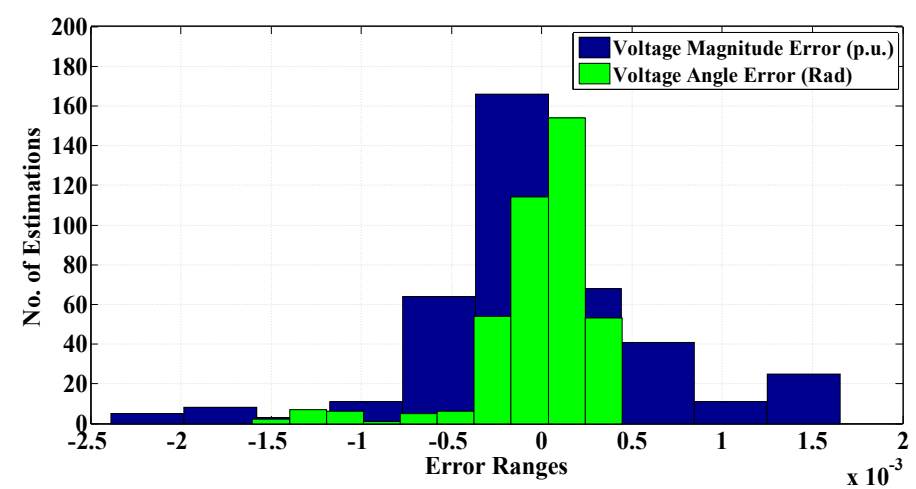

Fig. 11. Histograms of voltage phasor estimation errors in the weakly meshed operation mode and high DGs penetration level

\subsection{State estimation with different number of $\mu \mathrm{PMUs}$}

Unlike classical state estimation methods, observability of the entire network is not a prerequisite for our state estimation algorithm. Therefore, we install only $7 \mu$ PMUs along the test case to monitor a few buses in the system and do sensitivity analysis to assess the performance of our technique by considering different number of $\mu$ PMUs. The MAEs for voltage magnitude and angle estimation are shown in Table III. As shown, the mean absolute error of estimated voltage magnitudes and angles falls within $10^{-3}$ when $4 \mu \mathrm{PMUs}$ are installed. Therefore, we need at least $5 \mu$ PMUs in the system to keep the error indices within the acceptable rang of $10^{-4}$. The measurement at the remote-terminal bus is critical for the proposed DSSE method. If this measurement fails to communicate with the control center, the accuracy of the estimated states is degraded because the large injection current to the infeed point is not estimated accurately. For example, when the measurement at bus 1 in the test system is removed from the 7 measurements, and of the estimated states are and, respectively.

\section{TABLE III. STATE ESTIMATION PERFORMANCE WITH DIFFERENT NUMBER OF MPMUS}

No. of $\mu$ PMUs $\mu \mathrm{PMU}$ buses 


\begin{tabular}{c|c|c|c}
\hline 6 & $1,34,50,72,90,110$ & $5.63 \times 10^{-4}$ & $1.67 \times 10^{-4}$ \\
\hline 5 & $1,34,50,90,110$ & $5.78 \times 10^{-4}$ & $2.39 \times 10^{-4}$ \\
\hline 4 & $1,50,90,110$ & $1.10 \times 10^{-3}$ & $7.52 \times 10^{-4}$ \\
\hline 3 & $1,90,110$ & $2.40 \times 10^{-3}$ & $1.70 \times 10^{-3}$ \\
\hline
\end{tabular}

\section{The IEEE 123-Bus System}

Three-, double-, and single-phase overhead lines and cables, four capacitor banks and four voltage regulators are used in the IEEE 123-bus distribution network to supply 85 unbalanced loads with various phasing [46]. Based on the theorem 1, for and, . Therefore, we assume that $7 \mu$ PMUs are located at buses \# 116, 48, 25, 64, 83, 89 and 101 to just measure the voltage phasors at those buses. For this test system, a value of $10^{-4}$ is also obtained for . Our DSSE algorithm also performs satisfactory for this system with and. In this system, bus \#116 is connected to the external grid. Therefore, injection currents in its corresponding phases 233-235 have significant values in comparison with existing phases in other buses as shown in Fig. 12. In addition, actual and estimated phase-to-ground voltages for the existing phases of all buses are shown in Fig. 13. There are 123 buses and 256 phase-to-ground voltages that are estimated with errors in the range of and. 


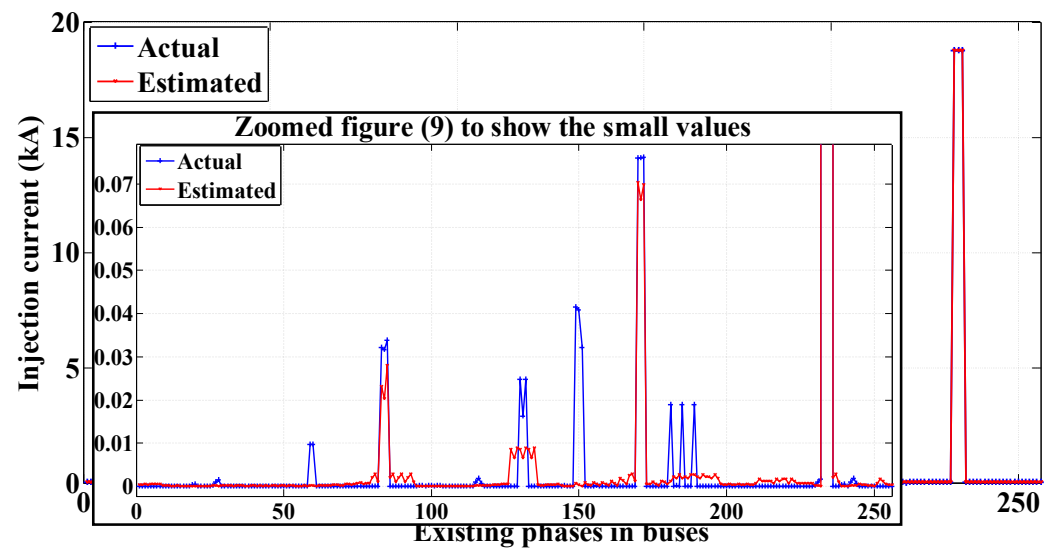

Fig. 12. Actual and estimated injection currents in the IEEE 123-bus system

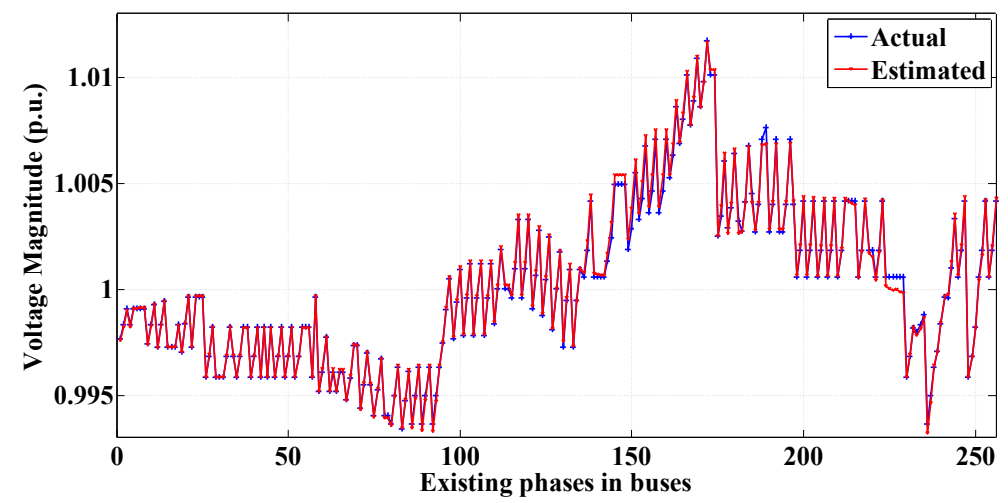

Fig. 13. Actual and estimated existing phase voltage magnitudes (p.u.) in the IEEE 123-bus system

\section{Discussion AND COMPARISON}

Distribution systems are not fully telemetered so far due to the huge cost of deploying the required infrastructure in thousands or tens of thousands of feeders. Therefore, pseudo measurements are not widely available in real distribution networks. For this reason, our DSSE algorithm using a few low-cost and highly accurate $\mu$ PMUs [26] has more practical benefits. Since our algorithm minimizes the $\ell^{1}$-norm of the current vector and not measurement errors, it provides a near-optimal solution with an acceptable accuracy. Actually, there is a trade-off between the measurements redundancy and the DSSE accuracy. If it is assumed that a distribution system is entirely monitored by redundant and enough accurate pseudo measurements like transmission networks, an 
optimal solution is obtained by solving the complex nonlinear and unbalanced optimization problems. For this situation, the WLS-based DSSE runs every few minutes due to the reporting rate of pseudo measurements. Note that our DSSE algorithm is executed every few seconds in parallel with WLS algorithm to provide the states of the system accurately during the gap between two sequential run.

In order to compare the proposed method with a conventional state estimator, a threephase weighted least square (WLS) estimator is developed to estimate the system states in the 134-bus system including 100 loads. The estimated voltage profiles in the phase (a) using WLS and the proposed method are shown in Fig. 14.
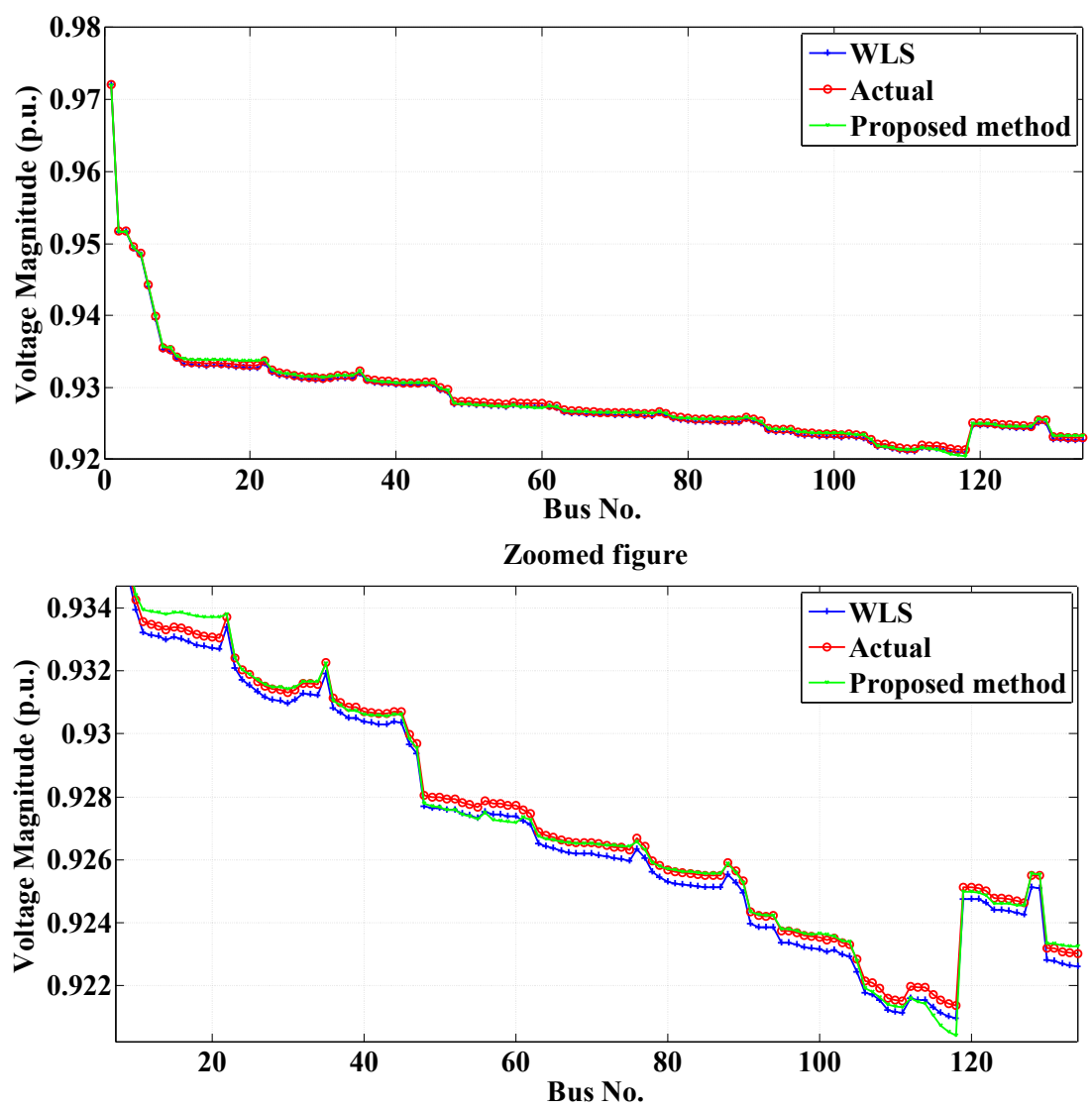

Fig. 14. Actual and estimated phase (a) voltage magnitudes (p.u.) in the 134-bus system using the proposed method and WLS technique 
It is assumed that all loads in the test case are monitored with smart meters which record the injection active and reactive powers with 3\% error [28]. Calculated and of the estimated states are and for the WLS method, respectively. These values are and for the proposed state estimator. Using the same computer, the computational time for WLS is $32 \mathrm{~s}$ that is significantly more than $3 \mathrm{~s}$ execution time for the proposed method. The and over the 50 simulation runs are shown in Fig. 15. These indices show that the proposed algorithm mostly outperforms the WLS method in voltage angle estimation, but the WLS performance is moderately better than the proposed method in estimating the voltage magnitudes.
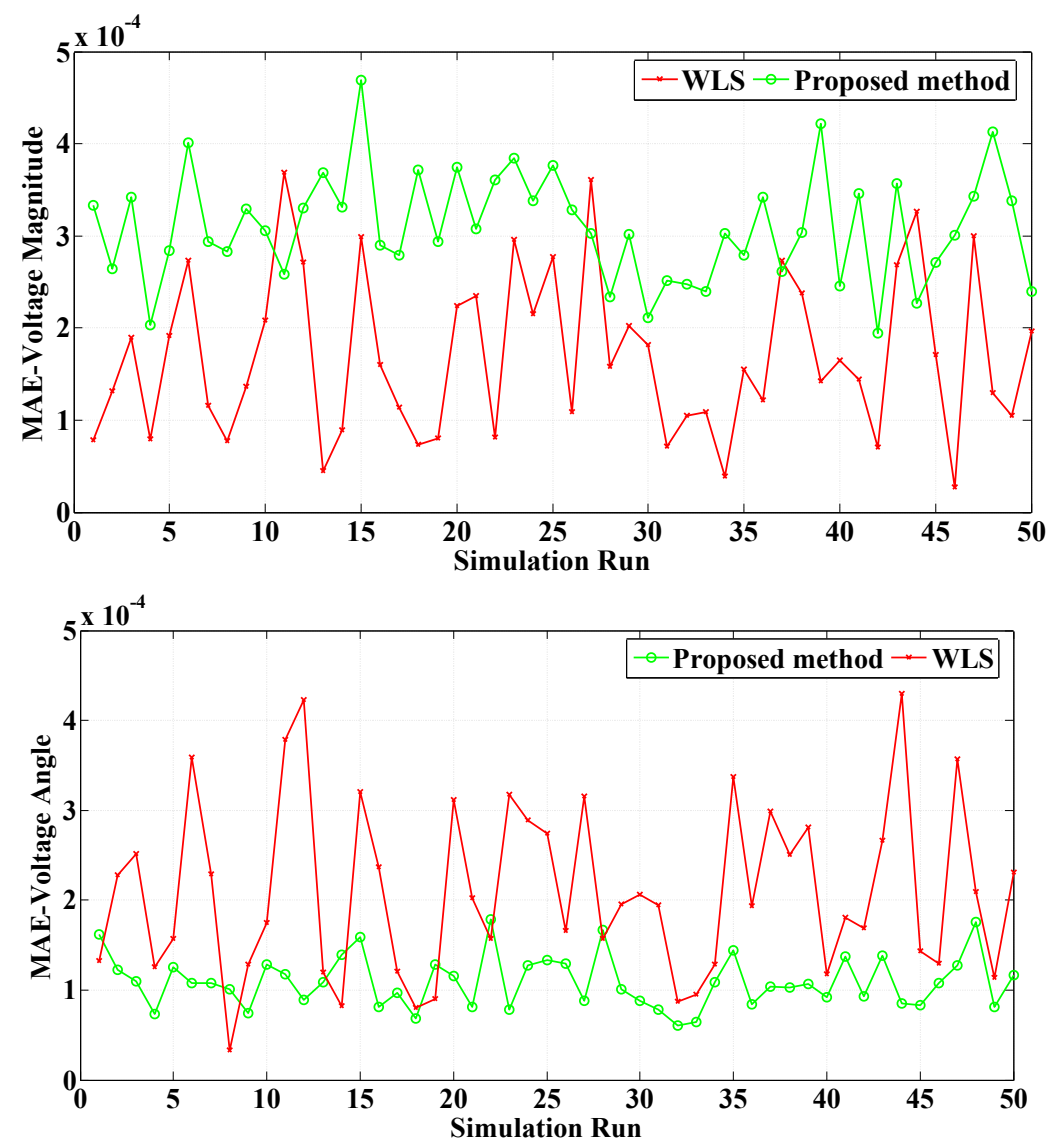

Fig. 15. and for the proposed method and WLS method over the 50 simulation runs

Since the available measurements including active/reactive powers are not linearly related to the system states, they cannot be directly used in the proposed methodology. 
However, there are some alternative solutions to integrate the proposed DSSE algorithm into classic state estimators for enhancing the estimated states accuracy.

- Integration into the post-processing step

In this approach, the estimated states by the proposed DSSE algorithm and the estimated states by classic WLS-based state estimators are used to provide a linear post-processing system to re-estimate the system states

where is the identity matrix, is the estimated complex voltage phasors, is the constant Jacobian matrix, and is the error vector of estimated states. Equation (15) is solved by the WLS method to re-estimate the system states vector whose accuracy is significantly improved compared to the accuracy. In the existing post-processing methods, the PMU measurements are just incorporated into the to re-estimate the. Therefore, and is the number of PMUs.

\section{- Deployment of a hybrid state estimator}

There is another scheme to improve the performance of WLS-based state estimators by adding the to the active/reactive power measurements in the iterative WLS algorithm steps

where is the active/reactive power measurement vector, is a nonlinear function relating power measurements to state variables and is the vector of power measurement errors. The state of the system is estimated by iteratively solving the WLS problem.

The proposed method can be also used as initialization for WLS methods or more 
complete ones that include all the available pseudo and AMI measurements.

\section{CONCLUSiOnS AND Future Works}

This paper proposes a novel three-phase distribution system state estimation (DSSE) method that only requires the knowledge of impedance-matrix of the network and the voltage phasors at few buses measured by micro-phasor measurement units ( $\mu$ PMUs). Unlike the earlier DSSE methods in the literature, only a few buses in distribution networks are observable by $\mu$ PMUs data and no injection power measurements are incorporated into our algorithm. The performance of the proposed DSSE algorithm is robust to noisy $\mu \mathrm{PMU}$ measurements, different DGs penetration levels, heterogeneity of overhead lines and weakly meshed operation mode in distribution networks. The performance of the algorithm is within the acceptable range even with less number of $\mu$ PMUs. This makes our algorithm resilient under the emergency conditions where a couple of $\mu$ PMUs fails to transmit their measured data to control center due to harsh weather condition or cyber-attacks. Also, power distribution companies can use our method to establish SE module with the minimum required number of $\mu$ PMUs and increase the number to enhance the estimation accuracy.

In this paper, $\mu$ PMUs are placed in distribution networks based on a generalized placement procedure. For future work, we will investigate the optimum $\mu \mathrm{PMU}$ placement strategy for distribution networks to obtain minimum level of estimation errors. In addition, although PMU measurements tend to be more accurate than pseudo measurements, they may also be contaminated by bad data. We will study bad data detection and identification in our future research. 


\section{REFERENCES}

1. F. C. Schweppe and J. Wildes, "Power system static-state estimation, Part I: Exact model," IEEE Trans. Power App. Syst., vol. PAS-89, no. 1, pp. 120-125, Jan. 1970.

2. F. F. Wu and A. F. Neyer, "Asynchronous distributed state estimation for power distribution systems," in Proc. 10th Power Syst. Comput. Conf., Aug. 1990, pp. 439-446.

3. I. Roytelman and S. M. Shahidehpour, "State estimation for electric power distribution systems in quasi real-time conditions," IEEE Trans. Power Del., vol. 8, no. 4, pp. 2009-2015, Oct. 1993.

4. C. N. Lu, J. H. Teng, andW. H. Liu, "Distribution system state estimation," IEEE Trans. Power Syst., vol. 10, no. 1, pp. 229-240, Feb. 1995.

5. M. E. Baran and A. W. Kelley, "A branch-current-based state estimation method for distribution systems," IEEE Trans. Power Syst., vol. 10, no. 1, pp. 483-491, Feb. 1995.

6. Y. Deng, Y. He, and B. Zhang, "A branch-estimation-based state estimation method for radial distribution systems," IEEE Trans. Power Del., vol. 17, no. 4, pp. 1057-1062, Oct. 2002.

7. W. M. Lin and J. H. Teng, "Distribution fast decoupled state estimation by measurement pairing," Proc. Inst. Elect. Eng., Gen., Transm., Distrib., vol. 143, no. 1, pp. 43-48, Jan. 1996.

8. K. Li, "State estimation for power distribution system and measurement impacts," IEEE Trans. Power Syst., vol. 11, no. 2, pp. 911-916, May 1996.

9. R. Singh, B. C. Pal, and R. A. Jabr, "Distribution system state estimation through Gaussian mixture model of the load as pseudo-measurement," IET Gen., Transm., Distrib., vol. 4, no. 1, pp. 50-59, January 2010 .

10. N. C.Woolley and J. V.Milanovic, "Statistical estimation of the source and level of voltage unbalance in distribution networks," IEEE Trans. Power Del., vol. 27, no. 3, pp. 1450-1460, Jul. 2012.

11. J. Wu, Y. He, and N. Jenkins, "A robust state estimator for medium voltage distribution networks," IEEE Trans. Power Syst., vol. 28, no. 2, pp. 1008-1016, May 2013.

12. I. Dzafic, H. T. Neisius, and S. Henselmeyer, "Real time distribution system state estimation based on interior point method," in Proc. 17th Power Syst. Computation Conf., Stockholm, Sweden, 2011, pp. 1-9.

13. P.M.De Oliveira-De Jesus and A.A.Rojas Quintana, "Distribution System State Estimation Model Using a Reduced Quasi-Symmetric Impedance Matrix," IEEE Trans. Power Syst., Early Access.

14. C.Muscas, M.Pau, P.A. Pegoraro, S.Sulis, F.Ponci, A.Monti, "Multiarea Distribution System State Estimation," IEEE Trans. Instrum. Meas., Early Access.

15. A. K. Ghosh, D. L. Lubkeman, and R. H. Jones, "Load modeling for distribution circuit state estimation," IEEE Trans. Power Del., vol. 12, pp. 999-1005, Apr. 1996.

16. E. Manitsas, R. Singh, B. C. Pal, and G. Strbac, "Distribution system state estimation using an artificial neural network approach for pseudo measurement modeling," IEEE Trans. Power Syst., vol. 27, no. 4, pp. 1888-1896, Nov. 2012.

17. V.Miranda, J. Pereira, and J. T. Saraiva, "Load allocation in DMS with a fuzzy state estimator," IEEE Trans. Power Syst., vol. 15, no. 2, pp. 529-534, May 2000.

18. B.P.Hayes, J.K.Gruber, M.Prodanovic, "A Closed-Loop State Estimation Tool for MV Network Monitoring and Operation," IEEE Trans. Smart Grid, Early Access.

19. J. Wu, Y. He, and N. Jenkins, "A robust state estimator for medium voltage distribution networks," IEEE Trans. Power Syst., vol. 28, no. 2, pp. 1008-1016, May 2013.

20. I. Dzafic, M. Gilles, R. A. Jabr, B. C. Pal, and S. Henselmeyer, "Real time estimation of loads in radial and unsymmetrical three-phase distribution networks," IEEE Trans. Power Syst., vol. 28, no. 4, pp. 4839-4848, Nov. 2013.

21. I. Dzafic, R. A. Jabr, I. Huseinagic, B. C. Pal, "Multi-Phase State Estimation Featuring IndustrialGrade Distribution Network Models," IEEE Trans. Smart Grid., Early Access, pp.1-10, http:// dx.doi.org/10.1109/TSG.2016.2592978.

22. A. P. S. Meliopoulos and F. Zhang, "Multiphase power flow and state estimation for power distribution systems," IEEE Trans. Power Syst., vol. 11, no. 2, pp. 939-946, May 1996.

23. D. A. Haughton and G. T. Heydt, "A linear state estimation formulation for smart distribution systems,” IEEE Trans. Power Syst., vol. 28, no. 2, pp. 1187-1195, May 2013.

24. M. Majidi, A. Arabali, and M. Etezadi-Amoli, "Fault Location in Distribution Networks by Compressive Sensing," IEEE Trans. Power Del., vol. 30, no. 4, pp. 1761-1769, Aug. 2015.

25. M. Majidi, M. Etezadi-Amoli, and M. S. Fadali, "A Novel Method for Single and Simultaneous Faults Location in Distribution Networks," IEEE Trans. Power Syst., vol. 30, no. 6, pp. 3368-3376, Nov. 2015.

26. A. von Meier, D. Culler, A. McEachen, and R. Arghandeh, "Micro-synchrophasors for distribution systems," in Proceedings of IEEE Innovative Smart Grid Technologies Conference (ISGT2014), 2014. 
27. Leite, Jônatas Boás, and José Roberto Sanches Mantovani. "Distribution System State Estimation Using the Hamiltonian Cycle Theory." IEEE Transactions on Smart Grid 7.1 (2016): 366-375.

28. J. Liu, J. Tang, F. Ponci, A. Monti, C. Muscas, and P. A. Pegoraro, "Trade-offs in PMU deployment for state estimation in active distribution grids," IEEE Trans. Smart Grid, vol. 3, no. 2, pp. 915-924, Jun. 2012.

29. M. Pau, P. A. Pegoraro, and S. Sulis, "Efficient branch-current-based distribution system state estimation including synchronized measurements," IEEE Trans. Instrum. Meas., vol. 62, no. 9, pp. 2419-2429, Sep. 2013.

30. Guo, Ye, et al. "A distributed state estimation method for power systems incorporating linear and nonlinear models." International Journal of Electrical Power \& Energy Systems 64 (2015): 608-616.

31. Chen, Xuebing, King Jet Tseng, and Gehan Amaratunga. "State estimation for distribution systems using micro-synchrophasors." Power and Energy Engineering Conference (APPEEC), 2015 IEEE PES Asia-Pacific. IEEE, 2015.

32. Roy, Ayindrila, Jitendranath Bera, and Gautam Sarkar. "Feed-forward State Estimation of Multi-bus Distribution Network Using Sample Shifting Technique." Electric Power Components and Systems (2016): 1-11.

33. Sarri, Styliani, et al. "Performance assessment of linear state estimators using synchrophasor measurements." IEEE Transactions on Instrumentation and Measurement 65.3 (2016): 535-548.

34. L. Zhan, J. Zhao, S. Gao, J. Cullis, Y. Liu, and Y. Liu, "Universal grid analyzer design and development," in Proc. 2015 IEEE Power Eng. Soc. Gen. Meet, pp. 1-5.

35. A.Gomez-Exposito, C.Gomez-Quiles, I.Dzafic, "State Estimation in Two Time Scales for Smart Distribution Systems," IEEE Trans. Smart Grid, vol. 6, no. 1, pp. 421-430, January 2015.

36. M. Majidi, M. S. Fadali, M. Etezadi-Amoli, and M. Oskuoee, "Partial Discharge Pattern Recognition via Sparse Representation and ANN," IEEE Trans. Dielectr. Electr. Insul., vol. 22, no. 2, pp. 1061-1070, April 2015.

37. S. M. Shafiul Alam, B. Natarajan, and A. Pahwa, "Distribution Grid State Estimation from Compressed Measurements," IEEE Trans. Smart Grid, vol. 5, no. 4, pp.1631-1642, July 2014.

38. H. Zhu and G. B. Giannakis, "Sparse overcomplete representations for efficient identification of power line outages," IEEE Trans. Power Syst., vol. 27, no. 4, pp. 2215-2224, Nov. 2012.

39. M. Majidi, M. Etezadi-Amoli, and M. S. Fadali, "A Sparse-Data-Driven Approach for Fault Location in Transmission Networks" IEEE Trans. Smart Grid, Early Access.

40. M. Babakmehr, M.G. Simoes, and Ahmed Al-durra. "Compressive Sensing for Smart Grid Security and Reliability", to be appeared in Saifur. Rahman and S.M. Muyeen, editor "Communication, Control and Security for the Smart Grid", Ch:13, IET, London, UK, 2016.

41. M. Babakmehr, M.G. Simoes, M.B. Wakin, F. Harirchi. "Compressive Sensing-based Smart Grid Topology Identification” IEEE Transaction on Industrial Informatics, vol. 12, no. 2, pp: 532-543, APRIL 2016.

42. M. Babakmehr, M.G. Simoes, M.B. Wakin, A. Al Durra and F. Harirchi, "Sprase-based Smart Grid Topology Identification", IEEE Trans on Industry Applications, doi: 10.1109/TIA. 2016.2574767[dx.doi.org].

43. M. Babakmehr, M.G. Simoes, A. Al-Durra, F. Harirchi, Q. Han. "Application of Compressive Sensing for Distributed and Structured Power Line Outage Detection in Smart Grids", in Proc with IEEE American Control Conference ACC 2015, pp 3682-3689, Chicago, Illinois, USA, July 2015.

44. M. Babakmehr, M.G. Simoes, M.B. Wakin, A. Al Durra, and F. Harirchi, "Smart grid topology identification using sparse recovery," in Proc. IEEE IAS Annu. Meeting, Oct. 2015, pp. 1-8, Dallas, USA.

45. J. Yang, et al, Performance Analysis of Sparse Recovery Models for Bad Data Detection and State Estimation in Electric Power Networks. 2016 IEEE Power and Energy Society General Meeting, Boston, MA, 2016, [Online]. Available: https://arxiv.org/ftp/arxiv/papers/1606/1606.02066.pdf.

46. Feeders, Distribution Test. "IEEE PES Distribution System Analysis Subcommittee." OnlineAvailable: http://www. ewh. ieee. org/soc/pes/dsaco m/testfeeders/index. html (2011).

47. E. Candes and M. Wakin, "An introduction to compressive sampling," IEEE Signal Processing Mag., vol. 25, no. 2, pp. 21-30, Mar. 2008.

48. S. J. Kim, K. Koh, M. Lustig, S. Boyd, and D. Gorinevsky, “An Interior-Point Method for LargeScale 1'-Regularized Least Squares," IEEE Journal on Selected Topics in Signal Processing, vol.1, no.4, pp.606-617, December 2007.

49. N. Z. Shor, "Minimization methods for non-differentiable functions," Springer Series in Computational Mathematics, 1985, Springer. 
50. The MOSEK Optimization Tools Version 2.5. User's Manual and Reference 2002 [Online]. Available: www.mosek.com, MOSEK ApS.

51. S. Chen, D. Donoho, and M. Saunders, "Atomic decomposition by basis pursuit," SIAM Rev., vol. 43, no. 1, pp. 129-159, 2001.

52. D. Donoho and Y. Tsaig, "Fast solution of 11 -norm minimization problems when the solution may be sparse," Manuscript 2006 [Online]. Available: http://www.stanford.edu/

53. J. Friedman, T. Hastie, and R. Tibshirani, "Regularization paths for generalized linear models via coordinate descent," J. Stat. Software, vol. 33, no. 1, pp. 1-22, Feb. 2010.

54. DIgSILENT, [online] Availabe: http://www.digsilent.de/

55. IDM Multifunction power system monitor. Available [online]: http://www.qualitrolcorp.com/ uploadedFiles/Siteroot/Products/IP-F23-02A-01E_IDM+(3).pdf

\section{ACKNOWLEDGEMENT}

This paper is based upon work supported by the National Science Foundation under

Grant No. IIA-1301726. 